\title{
Survival prediction for patients with non-resectable intrahepatic cholangiocarcinoma undergoing chemotherapy: a retrospective analysis comparing the tumor marker CA 19-9 with cross-sectional imaging
}

\author{
Felix Hahn ${ }^{1} \cdot$ Lukas Müller $^{1} \cdot$ Florian Jungmann $^{1} \cdot$ Aline Mähringer-Kunz ${ }^{1} \cdot$ Yasemin Tanyildizi $^{2} \cdot$ Christoph Düber $^{1}$. \\ Peter R. Galle ${ }^{3}$. Arndt Weinmann ${ }^{3,4} \cdot$ Roman Kloeckner $^{1}$ D
}

Received: 6 January 2020 / Accepted: 24 March 2020 / Published online: 30 March 2020

(c) The Author(s) 2020

\begin{abstract}
Purpose Carbohydrate antigen (CA) 19-9 has been established as the main serum marker for patients with intrahepatic cholangiocarcinoma (ICC). The aim of this study was to compare the prognostic value of CA 19-9 changes versus response determined by imaging in patients with ICC undergoing chemotherapy.

Methods Between 2003 and 2018, 151 patients with histopathologically confirmed ICC underwent chemotherapy at our tertiary care center for non-resectable or recurrent ICC, of whom 121 were included in this study. Serum CA 19-9 levels and imaging were retrospectively evaluated during chemotherapy. Log-rank testing and optimal stratification were used to classify patients into risk groups.

Results Prior to chemotherapy, baseline serum CA 19-9 levels above the previously published cut-off of $37 \mathrm{U} / \mathrm{ml}$ were associated with poor survival (median OS 8.7 vs. 12.4 months, $p=0.003$ ). After the beginning of chemotherapy, an increase in CA 19-9 of more than $40 \mathrm{U} / \mathrm{ml}$ resulted in impaired residual survival (median OS 5.0 vs. 12.1 months, $p<0.001$ ). However, progressive disease at the first follow-up imaging proved the strongest predictor for poor outcome (median OS 4.6 vs. 15.5 months, $p<0.001)$. In contrast to prior studies, our data did not show statistically relevant differences in survival time with respect to absolute or relative decreases in serum CA 19-9 levels.

Conclusion In our study, the disease control rate — that is, the absence of progressive disease-was the strongest predictor of prolonged residual OS. To this end, both CA 19-9 changes and progressive disease on initial follow-up showed remarkable discriminatory power, with the latter slightly outperforming the former. Therefore, imaging should remain the mainstay of patient evaluation during follow-up.
\end{abstract}

Keywords Intrahepatic cholangiocarcinoma $\cdot$ Chemotherapy $\cdot$ Survival prediction $\cdot$ RECIST 1.1

Electronic supplementary material The online version of this article (https://doi.org/10.1007/s00432-020-03200-2) contains supplementary material, which is available to authorized users.

Roman Kloeckner

roman.kloeckner@unimedizin-mainz.de

1 Department of Diagnostic and Interventional Radiology, University Medical Center of the Johannes GutenbergUniversity Mainz, Langenbeckst. 1, 55131 Mainz, Germany

2 Department of Neuroradiology, University Medical Center of the Johannes Gutenberg-University Mainz, Mainz, Germany
Department of Internal Medicine, University Medical Center of the Johannes Gutenberg-University Mainz, Mainz, Germany

4 Clinical Registry Unit (CRU), University Medical Center of the Johannes Gutenberg-University Mainz, Mainz, Germany 


\section{Introduction}

Intrahepatic cholangiocarcinoma (ICC) is the secondmost common primary liver malignancy. Its incidence has increased markedly over the past three decades and is estimated to be around $0.4-2.0 / 100,000$ in the low endemic western countries (Hahn et al. 2011; Petrick et al. 2016; Shaib et al. 2004; Yang et al. 2012).

Unfortunately for the affected patients, this tumor type is often at an advanced stage at the time of diagnosis. Resection is the only curative treatment option (Guro et al. 2017). Even if resection is possible, the recurrence rate is high and recurrence is reported in up to two-thirds of patients (Park et al. 2016). For patients for whom resection is either initially or in the course of the disease no longer possible, different chemotherapy regimens have been proposed. Since the publication of the multicenter UK-ABC 02 study in 2010, the combination of gemcitabine and cisplatin has been widely administered as first-line chemotherapy (Valle et al. 2010). Systemic chemotherapy is the mainstay of treatment, but in the last decade transarterial therapies such as transarterial chemo-embolization (TACE) and selective internal radiation therapy (SIRT) have also become treatment options for selected patients (Boehm et al. 2015). However, the prognosis for ICC patients remains poor (Weber et al. 2015).

Carbohydrate antigen (CA) 19-9 is the main serum tumor marker for patients with ICC (Brito et al. 2015). Besides having diagnostic value, CA 19-9 has repeatedly been shown to be useful in predicting the overall survival (OS) or progression-free survival of patients with ICC. However, there is disagreement about the optimal cut-off value that identifies patients with poor prognosis, with proposed cut-offs ranging from 37 to $1000 \mathrm{U} / \mathrm{ml}$ (Ali et al. 2007; Bolm et al. 2019; Coelho et al. 2017; He et al. 2018; Jiang et al. 2011). Regarding the influence of CA 19-9 changes on survival in patients undergoing chemotherapy, a literature search yielded only two studies that focused on this issue. The first, by Harder et al., postulates that a decrease in CA 19-9 under chemotherapy is prognostically favorable, without giving a specific cut-off value (Harder et al. 2007). In the second study, by Lee et al., a relative decrease in CA 19-9 of more than 50\% after two cycles of gemcitabine-based chemotherapy was associated with prolonged survival (Lee et al. 2016).

Apart from tumor markers, cross-sectional imaging is the standard of care to monitor treatment response in cancer patients. The response evaluation criteria in solid tumors (RECIST) objectively classifies response into complete response (CR), partial response (PR), stable disease (SD), and progressive disease (PD) (Eisenhauer et al. 2009). Moreover, imaging also provides information about whether progress is due to local tumor advancement or metastatic spread, and treatment adjustments can be initiated depending on imaging results.

The aim of this study was to compare the discriminatory power of CA 19-9 changes versus response determined by imaging to identify patients at risk while receiving palliative chemotherapy.

\section{Material and methods}

Between January 2003 and February 2018, 151 patients with histopathologically confirmed ICC were treated with chemotherapy for non-resectable or recurrent ICC at our tertiary care center. Follow-up ended on February 28, 2019. The patients were retrospectively identified out of a prospectively maintained clinical registry unit. Of the 151 patients, cross-sectional imaging prior to chemotherapy was missing in three patients. Baseline CA 19-9 values were missing in another 27 patients, and the remaining 121 patients were included in the study (Fig. 1). The study was approved by the responsible ethics committee (permit number 2018-13618).

Contrast-enhanced computed tomography or magnetic resonance imaging prior to and in the course of chemotherapy were evaluated to determine the size and number of intrahepatic lesions, translobar and extrahepatic tumor spread, and presence of nodal and distant metastases. Translobar spread was defined as tumor extension per

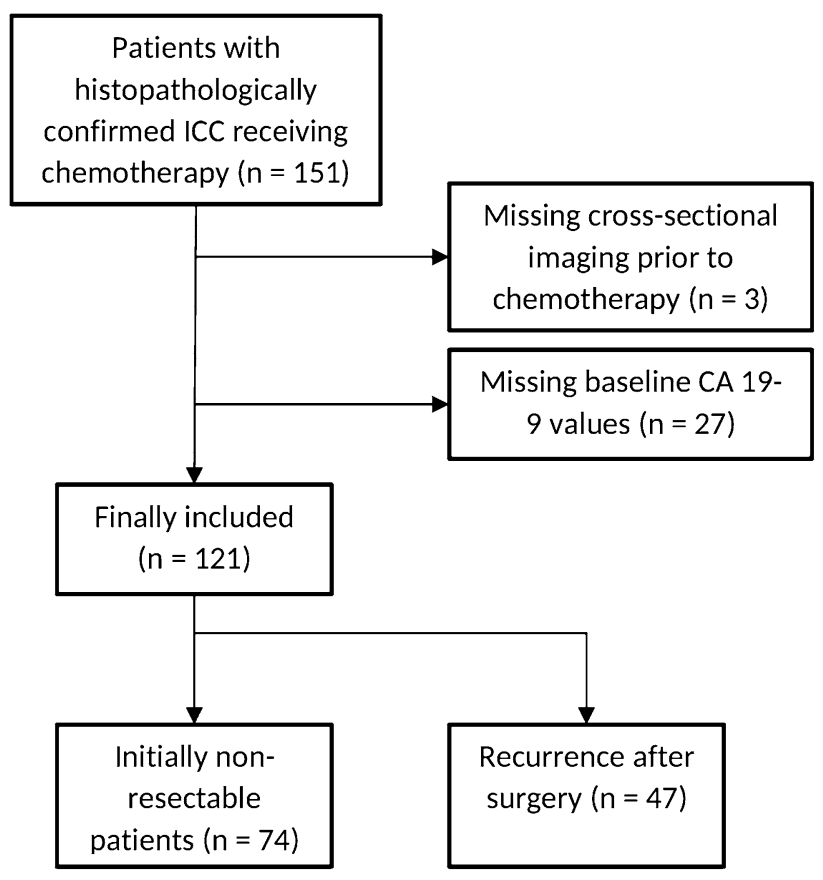

Fig. 1 STROBE flow chart showing the number of patients included in this study (CA 19-9, carbohydrate antigen 19-9) 
continuitatem or intrahepatic metastasis in more than one lobe. Extrahepatic spread was defined as a perforation of the liver viscera and/or continuous tumor infiltration in adjacent organs. The International Union against Cancer (UICC) staging system in its current 8th edition was used for tumor staging (Kim et al. 2017). Tumor response was determined according to the current RECIST guidelines (Eisenhauer et al. 2009) through independent examination by two boardcertified radiologists ( $\mathrm{FH}$ and $\mathrm{FJ}$ ), each with more than five years' experience in abdominal imaging. In case of discrepancy, a consensus reading was performed.

Serum CA 19-9 levels were collected prior to treatment and after initiation of chemotherapy from the laboratory information system. Data from follow-up visits were extracted from the hospital and radiology information systems. Death dates were queried at the appropriate Resident's Registration offices.

When investigating the relationship of baseline CA 19-9 values to survival time, the latter was calculated as the time elapsed from the beginning of chemotherapy to death or loss to follow-up. When investigating the predictive power of initial response under chemotherapy, the residual survival time from follow-up CA 19-9 value respectively follow-up imaging to death or loss to follow-up was calculated.

Statistical analyses were performed in R 3.6.1 (R Core Team 2019). Survival analyses were carried out using the packages "survival" and "survminer" (https://cran.r-proje ct.org/package=survival, https://cran.r-project.org/packa ge=survminer, accessed 30.11.2019). Log-rank testing was performed to stratify patients into survival groups. CA 19-9 cut-offs for dichotomization of the cohort were calculated using optimal stratification. Receiver operating characteristics (ROC) analysis was performed using the packages "pROC", "OptimalCutpoints", and "plotROC" (https:// cran.r-project.org/package $=$ pROC, https://cran.r-project.org/ package $=$ OptimalCutpoints, https://cran.r-project.org/packa ge=plotROC, accessed 30.11.2019).

As the intent of this analysis was exploratory, $p$-values should be interpreted in a descriptive manner. A $p$-value of $<0.05$ was considered statistically significant.

\section{Results}

\section{Baseline characteristics}

Median follow up time for all patients was 9.9 months. Administered chemotherapies were gemcitabine-based ( $n=86$, median number of cycles 5 ), capecitabine-based ( $n=9$, median number of cycles 6 ), fluorouracil-based $(n=8$, median number of cycles 12$)$, and others $(n=18)$. Further baseline characteristics are depicted in Table 1.
Table 1 Baseline characteristics of patients with intrahepatic cholangiocarcinoma (ICC) prior to systemic chemotherapy

\begin{tabular}{|c|c|}
\hline & All $(n=121)$ \\
\hline Age, years, median (IQR) & $63.1(54-71)$ \\
\hline \multicolumn{2}{|l|}{ Sex, $n(\%)$} \\
\hline Male & $66(54.5)$ \\
\hline Female & $55(45.5)$ \\
\hline Sum of intrahepatic lesions, mm, median (IQR) & $107(64-165)$ \\
\hline \multicolumn{2}{|l|}{ Tumor spread, $n(\%)$} \\
\hline Unifocal or intralobar metastases & $58(47.9)$ \\
\hline Translobar metastases & $50(41.3)$ \\
\hline Exclusively extrahepatic metastases & $13(10.8)$ \\
\hline \multicolumn{2}{|l|}{ Distant metastases, $n(\%)$} \\
\hline Negative & $43(35.5)$ \\
\hline Positive & $78(64.5)$ \\
\hline \multicolumn{2}{|l|}{ UICC stage, $n(\%)$} \\
\hline I & $11(9.1)$ \\
\hline II & $14(11.6)$ \\
\hline III & $18(14.9)$ \\
\hline IV & $78(64.4)$ \\
\hline CA 19-9 serum levels, U/ml, median (IQR) & $49(19-484)$ \\
\hline \multicolumn{2}{|l|}{ Previous therapies, $n(\%)$} \\
\hline Resection & $47(38.8)$ \\
\hline IAT & $9(7.4)$ \\
\hline None & $68(56.2)$ \\
\hline \multicolumn{2}{|l|}{ Chemotherapy regimen, $n(\%)$} \\
\hline Gemcitabine + cisplatin & $48(39.7)$ \\
\hline Other gemcitabine-based & $38(31.4)$ \\
\hline Capecitabine-based & $9(7.4)$ \\
\hline Fluorouracil-based & $8(6.6)$ \\
\hline Others & $18(14.9)$ \\
\hline \multicolumn{2}{|l|}{ Subsequent therapies, $n(\%)$} \\
\hline Second line chemotherapy & $41(33.9)$ \\
\hline IAT & $11(9.1)$ \\
\hline SBRT & $10(8.3)$ \\
\hline
\end{tabular}

$S D$ standard deviation, $I Q R$ interquartile range, UICC International Union against Cancer, $C A$ 19-9 carbohydrate antigen 19-9, IAT intraarterial therapy, $S B R T$ stereotactic body radiation therapy

\section{Baseline CA 19-9 values}

Baseline serum CA 19-9 levels above the previously published cut-off of $37 \mathrm{U} / \mathrm{ml}$ were associated with poor survival (median OS 8.7 vs. 12.4 months, $p=0.003$ ). However, all cut-offs from $22 \mathrm{U} / \mathrm{ml}$ to $2092 \mathrm{U} / \mathrm{ml}$ were predictive regarding OS and resulted in statistically significant divergence of survival curves. Using optimal stratification, the optimal cut-off was $60 \mathrm{U} / \mathrm{ml}$ in our cohort (median OS 8.1 vs. 11.5 months, $p<0.001$; Fig. 2). 

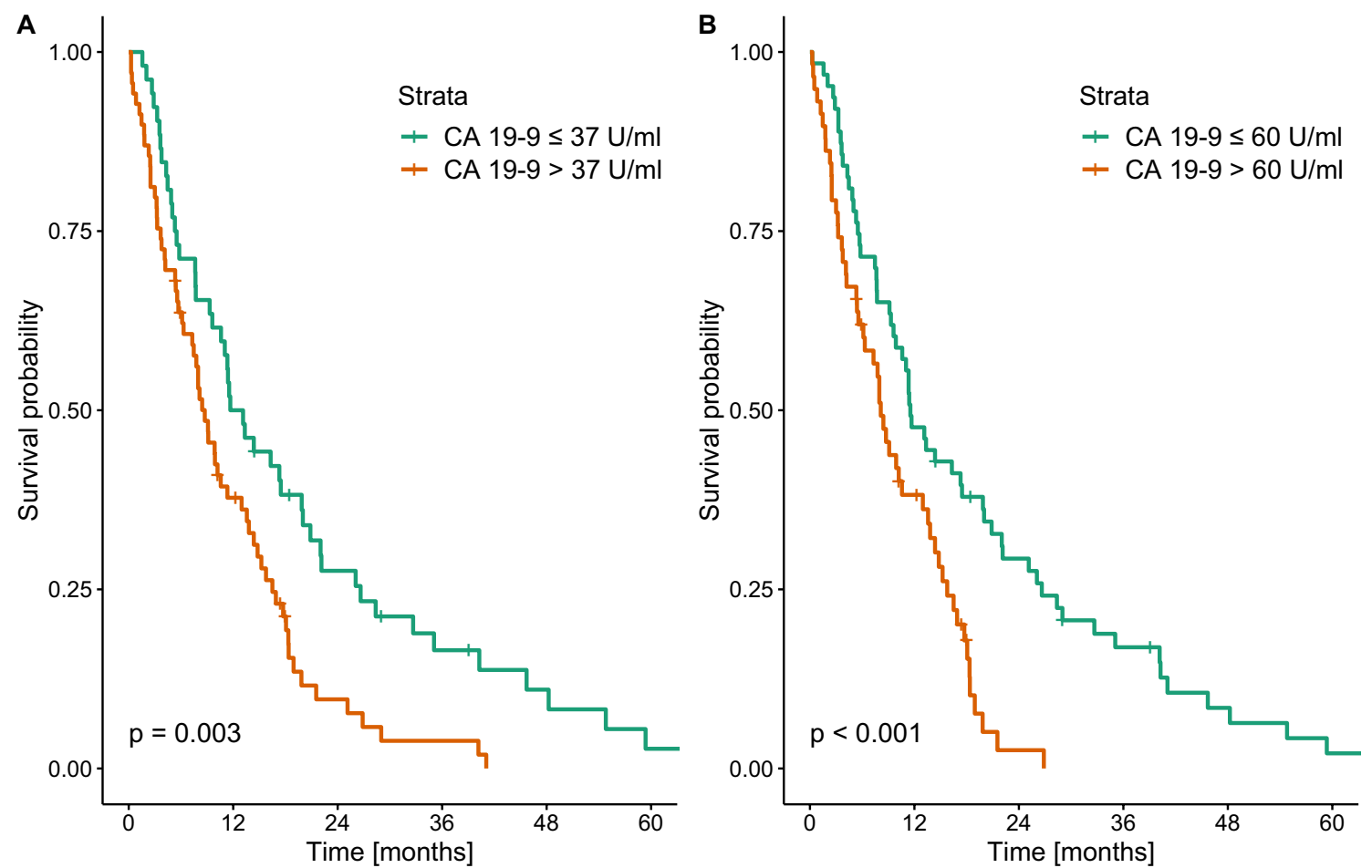

Number at risk

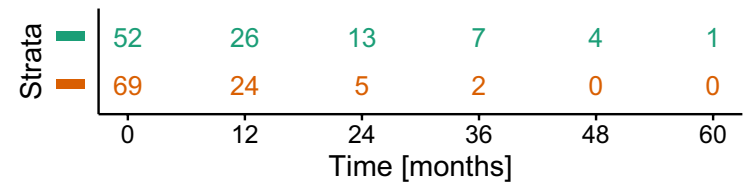

Number at risk

\begin{tabular}{|c|c|c|c|c|c|c|}
\hline \multirow{3}{*}{ 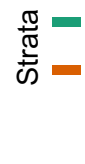 } & 63 & 30 & 17 & 9 & 4 & 1 \\
\hline & 58 & 20 & 1 & 0 & 0 & 0 \\
\hline & 0 & 12 & $\begin{array}{r}24 \\
\text { Tim }\end{array}$ & 36 & 48 & 60 \\
\hline
\end{tabular}

Fig. 2 Kaplan-Meier curves of overall survival using baseline CA 19-9 cut-offs at $37 \mathrm{U} / \mathrm{ml}$ (a) and $60 \mathrm{U} / \mathrm{ml}$ (b)

\section{Initial response to chemotherapy}

Serum CA 19-9 levels within 180 days after the start of chemotherapy were missing in 31 patients; 90 patients had follow-up values within that timeframe, with a median time between start of chemotherapy and first follow-up CA $19-9$ value of 60 days (interquartile range 40-91 days). An increase in CA 19-9>40 U/ml under chemotherapy was associated with significantly impaired residual survival using optimal stratification (median OS 5.0 vs. 12.1 months, $p<0.001$; Fig. 3a).

When investigating response in imaging, the median time between the start of chemotherapy and the first follow-up imaging was 66 days (interquartile range 49-90 days). In our cohort, the majority of patients achieved SD under chemotherapy $(n=52)$, followed by patients with PD $(n=32)$. Only a small percentage of patients actually achieved PR under chemotherapy $(n=8)$, and none achieved CR.

$\mathrm{PD}$ at the first follow-up investigation despite chemotherapy was associated with significantly worse residual
OS compared with patients with SD or PR (median OS 4.6 vs. 15.5 months, $p<0.001$; Fig. 3b). In case of initial tumor progression $(n=32), 11$ patients received second-line chemotherapy, 3 patients intraarterial therapies, 3 patients stereotactic body radiation therapy, and 7 patients were switched to best supportive care. Eight patients were initially continued on first-line therapy who had a rather constant tumor load and only radiographic evidence of small new metastases. Among the 29 patients who had no records of performed follow-up imaging, survival was even poorer, with a median survival of only 3.8 months after initiation of chemotherapy.

When using a combination of the above-mentioned factors and classifying patients with either progressive disease in imaging or an increase in CA 19-9 of more than $40 \mathrm{U} /$ $\mathrm{ml}$ as tumor progression, stratification of patients into risk groups only marginally improved compared to the monofactorial approach (median OS 4.6 months vs 15.7 months, $p<0.001$, Fig S1). 

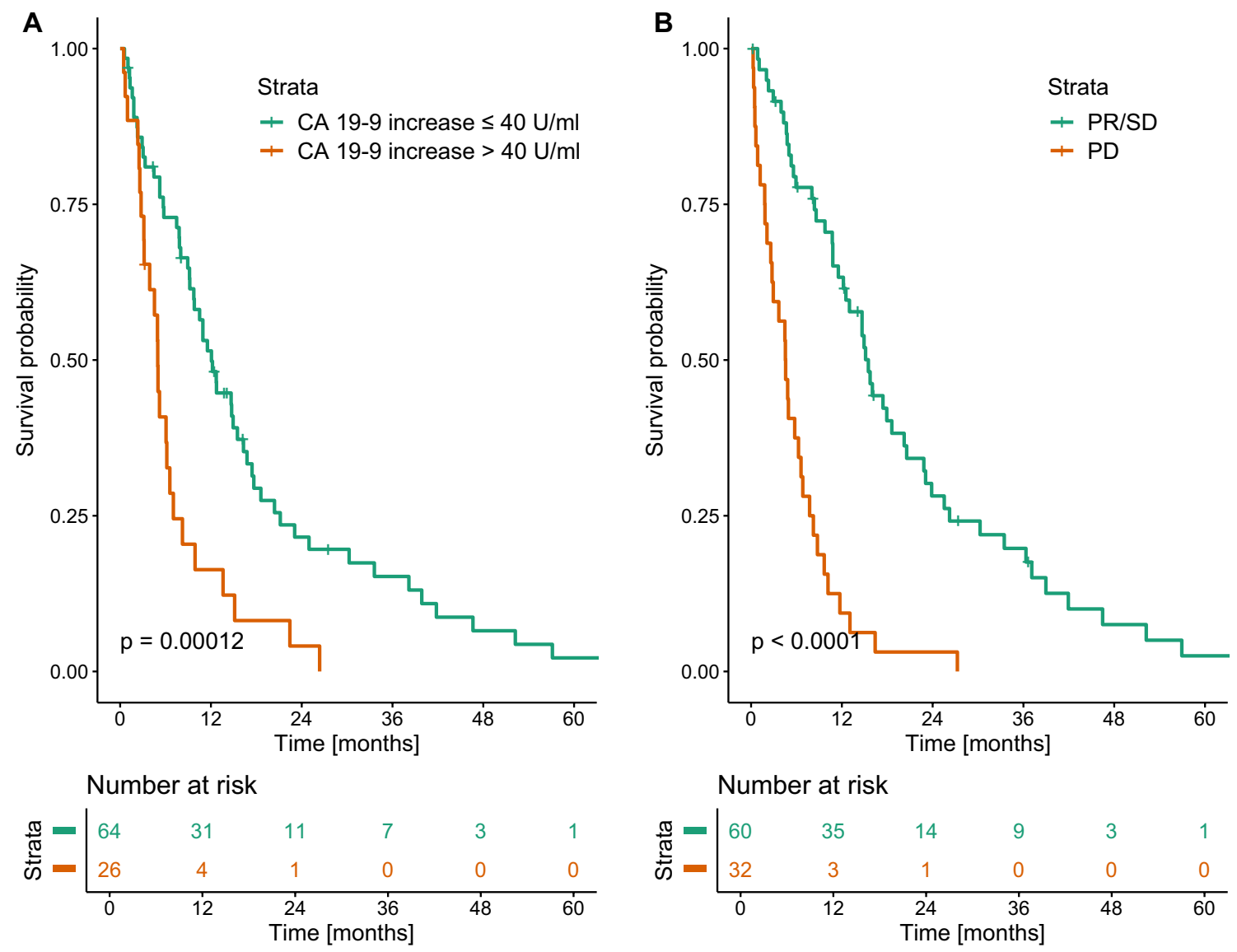

Fig. 3 Kaplan-Meier curves of overall survival after chemotherapy start stratified according to absolute CA 19-9 increases (a) und response in cross-sectional imaging (b) ( $P R$ partial response, $S D$ stable disease, $P D$ progressive disease)

\section{Correlation between CA 19-9 changes and imaging response}

After initiation of chemotherapy, follow-up serum CA 19-9 levels and imaging within 28 days of each another were available for 66 patients.

The results of ROC analysis investigating changes in serum CA 19-9 values with respect to progressive disease in follow-up imaging are depicted in Fig. 4 and yielded an area under the curve of 0.73 . The contingency table that resulted from using optimal cut-offs with regard to the positive and negative predictive values of changes in serum CA 19-9 level and imaging response is depicted in Table 2.

Here, an increase of CA 19-9 above $55 \mathrm{U} / \mathrm{ml}$ resulted in a positive predictive value for progressive disease in imaging of $76 \%$, whereas a decrease in CA $19-9$ of more than $2 \mathrm{U} / \mathrm{ml}$ resulted in a negative predictive value of $91 \%$. However, 27 of 66 patients $(41 \%)$ showed intermediate changes in serum CA 19-9 levels, from -2 to $55 \mathrm{U} / \mathrm{ml}$, with no clear association between CA 19-9 changes in this range and response in imaging.

\section{Discussion}

In our study, baseline serum CA 19-9 levels prior to chemotherapy were predictive of poor survival. During the course of chemotherapy, an initial increase in CA 19-9 outperformed baseline serum levels with regards to survival stratification. PD in follow-up imaging showed the best predictive performance towards residual OS in our cohort.

While several studies have previously shown prognostic value in baseline CA 19-9 serum levels with regard to survival (Ali et al. 2007; Bolm et al. 2019; Coelho et al. 2017; He et al. 2018; Jiang et al. 2011), studies investigating CA 19-9 kinetics in patients with ICC undergoing chemotherapy are scarce. A study by Harder et al. investigated 70 patients with biliary tract cancers, among them 33 patients with ICC. In their cohort, patients with a decrease in CA 19-9 levels after treatment showed an improved survival, without specifying a cut-off value (Harder et al. 2007). Lee et al. investigated a cohort of 179 patients, including 97 with ICC. They found a decline of at least 50\% in CA 19-9 level to be the most significant prognostic factor within their entire cohort, 
Fig. 4 ROC analysis comparing changes in serum CA 19-9 levels with progressive disease in follow-up imaging (AUC, area under the curve)

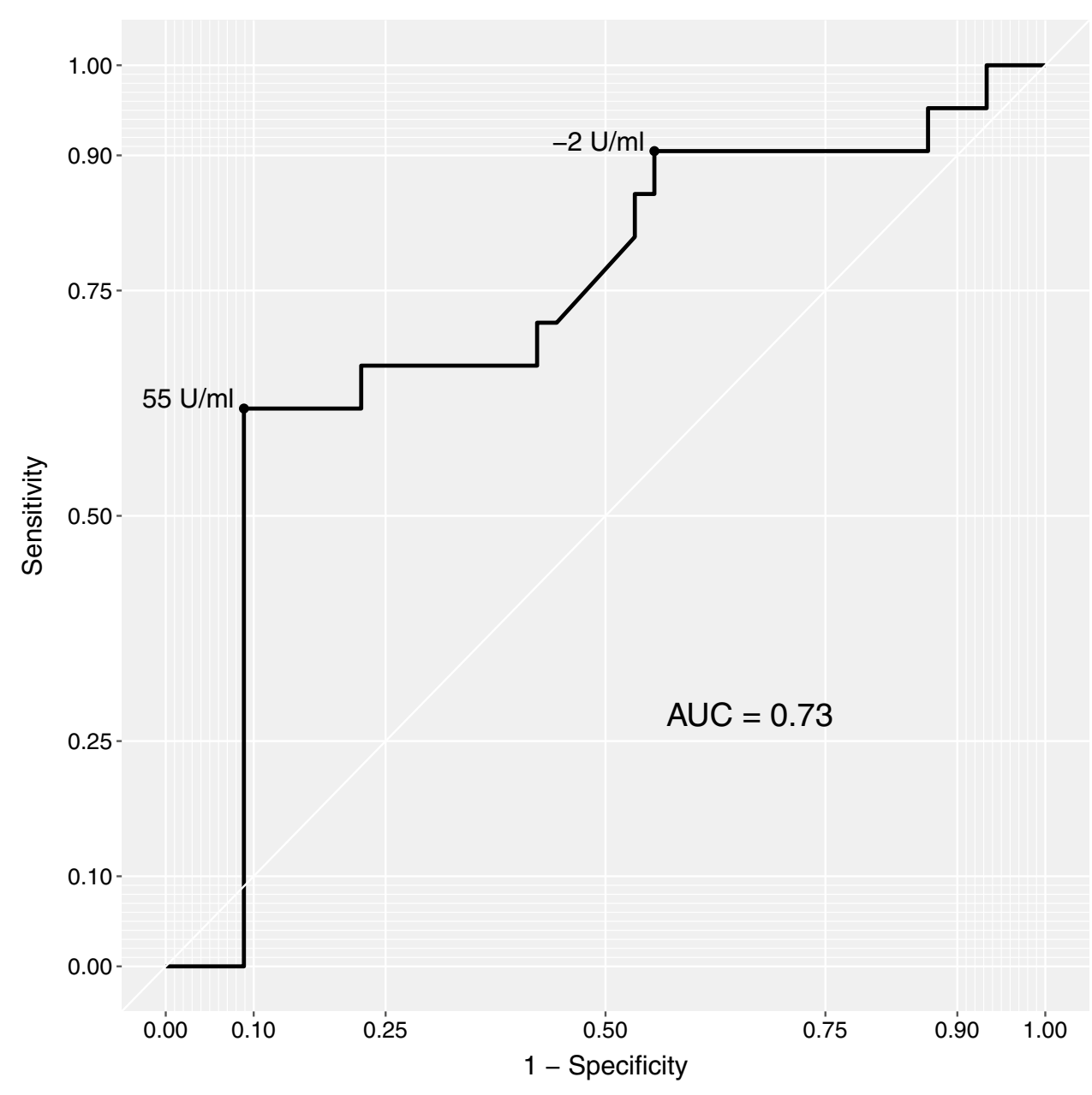

Table 2 Contingency table showing changes in serum CA 19-9 levels and response in cross-sectional imaging

\begin{tabular}{lcrrc}
\hline & PR & SD & PD & Sum \\
\hline CA 19-9 decrease $>2$ U/ml & 3 & 17 & 2 & 22 \\
CA 19-9 change between -2 to $55 \mathrm{U} / \mathrm{ml}$ & 3 & 18 & 6 & 27 \\
CA 19-9 increase $>55 \mathrm{U} / \mathrm{ml}$ & 0 & 4 & 13 & 17 \\
Sum & 6 & 39 & 21 & 66 \\
\hline
\end{tabular}

$P R$ partial response, $S D$ stable disease, $P D$ progressive disease

with a median survival time of 16.0 months vs. 9.0 months (Lee et al. 2016). Our data did not show statistically relevant differences in survival times with respect to absolute or relative decreases in serum CA 19-9 levels.

Previous studies have suggested that tumor location influences the effectiveness of chemotherapy, with patients affected by distal cholangiocarcinoma showing a larger benefit (Serafini et al. 2001), while the survival benefit of chemotherapy in the management of patients with ICC is limited (Rahnemai-Azar et al. 2017). In the study by Lee et al., perihilar and distal cholangiocarcinomas also showed lower hazard ratios in hazard regression analysis than ICC (Lee et al. 2016). This might explain the findings in our study, which focused exclusively on patients with ICC. In the studies by Harder and Lee, ICCs constituted only $47 \%$ and $54 \%$ of the tumors, respectively (Harder et al. 2007; Lee et al. 2016). Therefore, the better overall response to chemotherapy they observed might be due to the number of perihilar and distal cholangiocarcinomas.

In contrast to Harder et al. and Lee et al., we found that the disease control rate, comprising $\mathrm{CR}, \mathrm{PR}$, and $\mathrm{SD}$ - that is, the absence of progressive disease-was the strongest predictor of prolonged residual OS in our cohort. PD as the best predictor of poor survival in initial follow-up imaging has been observed in other aggressive tumor entities: Lara et al. investigated survival in patients with advanced nonsmall cell lung cancer under platinum-based chemotherapy, and found that differentiating between PD and the disease control rate at week 8 was the strongest predictor of subsequent survival outcome (Lara et al. 2008).

Moreover, the positive predictive value of increasing serum CA 19-9 levels and negative predictive values of decreasing serum CA 19-9 levels with regard to PD and 
the disease control rate in imaging response were $76 \%$ and $91 \%$, respectively. Therefore, laboratory markers have the potential to detect at-risk patients who are likely to benefit from closer imaging surveillance.

In our cohort, patients with CA 19-9 changes from -2 to $55 \mathrm{U} / \mathrm{ml}$ constituted a subgroup with heterogeneous imaging responses and progressive disease in $22 \%$ of patients. However, differentiating viable tumor tissue and tumor necrosis under chemotherapy can be challenging, especially in liver tumors, and thus imaging response may be misleading. This has led to the development of modified RECIST (mRECIST) for hepatocellular carcinoma and the Choi criteria for gastrointestinal stromal tumors (Choi et al. 2004; Lencioni et al. 2017). In the absence of a specific response evaluation system for ICC, we decided to use RECIST in its current version because of its widespread use (Eisenhauer et al. 2009). Despite these shortcomings, PD in follow-up imaging was the best predictor of residual OS in our study.

As intrahepatic cholangiocarcinoma is a rare tumor entity, the vast majority of studies investigating systemic chemotherapies are retrospective (Chun and Javle 2017). This holds true for our analysis as well, and the times at which CA 19-9 assays and imaging follow-ups were performed were not standardized. However, the median times between the start of chemotherapy and the first follow-up investigation were 60 and 66 days for CA 19-9 and imaging, respectively, corresponding to the end of the third cycle in the gemcitabine-based chemotherapy regimen (cycle length 21 days) (Valle et al. 2010).

CA 19-9 laboratory testing is less costly and more convenient than cross-sectional imaging; however, the information obtained by imaging evaluation exceeds the information obtained by the tumor marker considerably. Knowledge of the site of tumor progression has direct impact on further treatment options as it is essential to devise optimal treatment (Rizvi et al. 2018). Moreover, false-positive CA 19-9 values when diagnosing patients with cholangiocarcinoma have been reported in over 15\% of cases (Qin et al. 2004) and false positive CA 19-9 values due to concomitant inflammation or stenosis may lead to an increased distress in patients during follow-up.

Our analysis has several further limitations. First, the study was single-centered and conducted retrospectively. Second, the sample size $(n=121)$ was moderate. However, due to the low incidence of ICC in western countries, prior studies on the kinetics of ICC had to cope with similar or even considerably smaller sample sizes. Moreover, as gemcitabine in combination with cisplatin became the current standard in 2010, the cohort was heterogeneous in terms of chemotherapy regimens due to the long recruitment period. Multidisciplinary, multi-center efforts are needed in further studies to achieve larger cohorts and allow for prospective studies.

\section{Conclusion}

In our study, the disease control rate (the absence of progressive disease) was the strongest predictor of prolonged residual OS in our cohort. Changes in CA 19-9 values and progressive disease on initial follow-up both showed remarkable discriminatory power, with PD slightly outperforming CA 19-9. Therefore, imaging should remain the mainstay of patient evaluation during follow-up. However, changes in serum CA 19-9 levels have the potential to identify patients who are at risk for progressive disease and likely to benefit from closer imaging evaluation.

Acknowledgements Open Access funding provided by Projekt DEAL. FH was granted protected research time by a University Center for Tumor Diseases/Transmed Fellowship of the Johannes Gutenberg University Mainz Medical Center.

\section{Compliance with ethical standards}

Conflict of interest PRG has received grants and personal fees from Bayer and personal fees from Bristol-Myers Squibb, MSD Sharp \& Dohme, Lilly, Sillajen, SIRTEX, and AstraZeneca. AW has received speaker fees and travel grants from Bayer. RK has received speaker fees from BTG, Guerbet, and SIRTEX and personal fees from Boston Scientific, Bristol-Myers Squibb, Guerbet, and SIRTEX. None of these companies supported this study, and none of the authors reports a conflict of interest.

Ethics approval/trial number The study was approved by the responsible ethics committee (Ethics committee of the Medical Association of Rhineland Palatinate, Mainz, Germany) for the retrospective analysis of clinical data (permit number 2018-13618). Additional examinations were not performed. Patient records and information were anonymized and de-identified prior to analysis.

Open Access This article is licensed under a Creative Commons Attribution 4.0 International License, which permits use, sharing, adaptation, distribution and reproduction in any medium or format, as long as you give appropriate credit to the original author(s) and the source, provide a link to the Creative Commons licence, and indicate if changes were made. The images or other third party material in this article are included in the article's Creative Commons licence, unless indicated otherwise in a credit line to the material. If material is not included in the article's Creative Commons licence and your intended use is not permitted by statutory regulation or exceeds the permitted use, you will need to obtain permission directly from the copyright holder. To view a copy of this licence, visit http://creativecommons.org/licenses/by/4.0/.

\section{References}

Ali CW, Kaye TF, Adamson DJA, Tait IS, Polignano FM, Highley MS (2007) CA 19-9 and survival in advanced and unresectable pancreatic adenocarcinoma and cholangiocarcinoma. J Gastrointest Cancer 38:108-114. https://doi.org/10.1007/s 1202 9-008-9019-x

Boehm LM, Jayakrishnan TT, Miura JT, Zacharias AJ, Johnston FM, Turaga KK, Gamblin TC (2015) Comparative effectiveness of 
hepatic artery based therapies for unresectable intrahepatic cholangiocarcinoma. J Surg Oncol 111:213-220. https://doi. org/10.1002/jso.23781

Bolm L, Petrova E, Weitz J, Rückert F, Wittel UA, Makowiec F, Lapshyn H, Bronsert P, Rau BM, Khatkov IE, Bausch D, Keck T, Wellner UF, Distler M (2019) Prognostic relevance of preoperative bilirubin-adjusted serum carbohydrate antigen 19-9 in a multicenter subset analysis of 179 patients with distal cholangiocarcinoma. HPB (Oxford). https://doi.org/10.1016/j.hpb.2019.03.363

Brito AF, Abrantes AM, Encarnação JC, Tralhão JG, Botelho MF (2015) Cholangiocarcinoma: from molecular biology to treatment. Med Oncol 32:245. https://doi.org/10.1007/s12032-015-0692-x

Choi H, Charnsangavej C, de Castro Faria S, Tamm EP, Benjamin RS, Johnson MM, Macapinlac HA, Podoloff DA (2004) CT evaluation of the response of gastrointestinal stromal tumors after imatinib mesylate treatment: a quantitative analysis correlated with FDG PET findings. AJR Am J Roentgenol 183:1619-1628. https://doi. org/10.2214/ajr.183.6.01831619

Chun YS, Javle M (2017) Systemic and adjuvant therapies for intrahepatic cholangiocarcinoma. Cancer Control 24:1073274817729241. https://doi.org/10.1177/1073274817729241

Coelho R, Silva M, Rodrigues-Pinto E, Cardoso H, Lopes S, Pereira P, Vilas-Boas F, Santos-Antunes J, Costa-Maia J, Macedo G (2017) CA 19-9 as a marker of survival and a predictor of metastization in cholangiocarcinoma. GE Port J Gastroenterol 24:114-121. https ://doi.org/10.1159/000452691

Eisenhauer EA, Therasse P, Bogaerts J, Schwartz LH, Sargent D, Ford R, Dancey J, Arbuck S, Gwyther S, Mooney M, Rubinstein L, Shankar L, Dodd L, Kaplan R, Lacombe D, Verweij J (2009) New response evaluation criteria in solid tumours: revised RECIST guideline (version 1.1). Eur J Cancer 45:228-247. https://doi. org/10.1016/j.ejca.2008.10.026

Guro H, Kim JW, Choi Y, Cho JY, Yoon Y-S, Han H-S (2017) Multidisciplinary management of intrahepatic cholangiocarcinoma: Current approaches. Surg Oncol 26:146-152. https://doi. org/10.1016/j.suronc.2017.03.001

Hahn T, Ciesek S, Wegener G, Plentz RR, Weismüller TJ, Wedemeyer H, Manns MP, Greten TF, Malek NP (2011) Epidemiological trends in incidence and mortality of hepatobiliary cancers in Germany. Scand J Gastroenterol 46:1092-1098. https://doi. org/10.3109/00365521.2011.589472

Harder J, Kummer O, Olschewski M, Otto F, Blum HE, Opitz O (2007) Prognostic relevance of carbohydrate antigen 19-9 levels in patients with advanced biliary tract cancer. Cancer Epidemiol Biomarkers Prev 16:2097-2100. https://doi.org/10.1158/10559965.EPI-07-0155

He C, Zhang Y, Song Y, Wang J, Xing K, Lin X, Li S (2018) Preoperative CEA levels are supplementary to CA19-9 levels in predicting prognosis in patients with resectable intrahepatic cholangiocarcinoma. J Cancer 9:3117-3128. https://doi.org/10.7150/jca.25339

Jiang B-g, Ge R-1, Sun L-1, Zong M, Wei G-t, Zhang Y-j (2011) Clinical parameters predicting survival duration after hepatectomy for intrahepatic cholangiocarcinoma. Can J Gastroenterol 25:603-608

Kim Y, Moris DP, Zhang X-F, Bagante F, Spolverato G, Schmidt C, Dilhoff M, Pawlik TM (2017) Evaluation of the 8th edition American Joint Commission on Cancer (AJCC) staging system for patients with intrahepatic cholangiocarcinoma: A surveillance, epidemiology, and end results (SEER) analysis. J Surg Oncol 116:643-650. https://doi.org/10.1002/jso.24720
Lara PN, Redman MW, Kelly K, Edelman MJ, Williamson SK, Crowley JJ, Gandara DR (2008) Disease control rate at 8 weeks predicts clinical benefit in advanced non-small-cell lung cancer: results from Southwest Oncology Group randomized trials. J Clin Oncol 26:463-467. https://doi.org/10.1200/JCO.2007.13.0344

Lee BS, Lee SH, Son JH, Jang DK, Chung KH, Paik WH, Ryu JK, Kim Y-T (2016) Prognostic value of CA 19-9 kinetics during gemcitabine-based chemotherapy in patients with advanced cholangiocarcinoma. J Gastroenterol Hepatol 31:493-500. https://doi. org/10.1111/jgh.13059

Lencioni R, Montal R, Torres F, Park J-W, Decaens T, Raoul J-L, Kudo M, Chang C, Ríos J, Boige V, Assenat E, Kang Y-K, Lim H-Y, Walters I, Llovet JM (2017) Objective response by mRECIST as a predictor and potential surrogate end-point of overall survival in advanced HCC. J Hepatol 66:1166-1172. https://doi. org/10.1016/j.jhep.2017.01.012

Park HM, Yun SP, Lee EC, Lee SD, Han S-S, Kim SH, Park S-J (2016) Outcomes for patients with recurrent intrahepatic cholangiocarcinoma after surgery. Ann Surg Oncol 23:4392-4400. https://doi. org/10.1245/s10434-016-5454-2

Petrick JL, Braunlin M, Laversanne M, Valery PC, Bray F, McGlynn KA (2016) International trends in liver cancer incidence, overall and by histologic subtype, 1978-2007. Int J Cancer 139:15341545. https://doi.org/10.1002/ijc.30211

Qin X-L, Wang Z-R, Shi J-S, Lu M, Wang L, He Q-R (2004) Utility of serum CA19-9 in diagnosis of cholangiocarcinoma: in comparison with CEA. World J Gastroenterol 10:427-432. https:// doi.org/10.3748/wjg.v10.i3.427

R Core Team (2018) R: a language and environment for statistical computing. https://www.R-project.org/

Rahnemai-Azar AA, Weisbrod AB, Dillhoff M, Schmidt C, Pawlik TM (2017) Intrahepatic cholangiocarcinoma: current management and emerging therapies. Expert Rev Gastroenterol Hepatol 11:439-449. https://doi.org/10.1080/17474124.2017.1309290

Serafini FM, Sachs D, Bloomston M, Carey LC, Karl RC, Murr MM, Rosemurgy AS (2001) Location, not staging, of cholangiocarcinoma determines the role for adjuvant chemoradiation therapy. Am Surg 67:839-843

Shaib YH, Davila JA, McGlynn K, El-Serag HB (2004) Rising incidence of intrahepatic cholangiocarcinoma in the United States: a true increase? J Hepatol 40:472-477. https://doi.org/10.1016/j. jhep.2003.11.030

Valle J, Wasan H, Palmer DH, Cunningham D, Anthoney A, Maraveyas A, Madhusudan S, Iveson T, Hughes S, Pereira SP, Roughton M, Bridgewater J (2010) Cisplatin plus gemcitabine versus gemcitabine for biliary tract cancer. N Engl J Med 362:1273-1281. https ://doi.org/10.1056/NEJMoa0908721

Weber SM, Ribero D, O’Reilly EM, Kokudo N, Miyazaki M, Pawlik TM (2015) Intrahepatic cholangiocarcinoma: expert consensus statement. HPB (Oxford) 17:669-680. https://doi.org/10.1111/ hpb. 12441

Yang JD, Kim B, Sanderson SO, Sauver JS, Yawn BP, Larson JJ, Therneau TM, Roberts LR, Gores GJ, Kim WR (2012) Biliary tract cancers in Olmsted County, Minnesota, 1976-2008. Am J Gastroenterol 107:1256-1262. https://doi.org/10.1038/ ajg.2012.173

Publisher's Note Springer Nature remains neutral with regard to jurisdictional claims in published maps and institutional affiliations. 